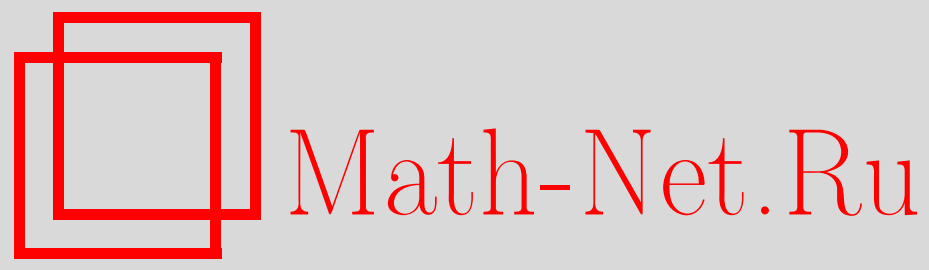

А. Ю. Плахов, Предельное поведение спектра в одном классе больших случайных матриц, Функи. анализ и его прил., 1997, том 31, выпуск 2, 85-88

DOI: https://doi.org/10.4213/faa467

Использование Общероссийского математического портала MathNet.Ru подразумевает, что вы прочитали и согласны с пользовательским соглашением

http://www . mathnet.ru/rus/agreement

Параметры загрузки:

IP : 34.227 .88 .159

26 апреля 2023 г., 13:19:47

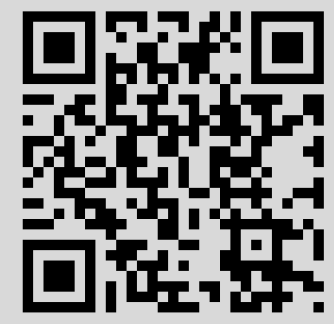


В силу (3) равенство (2) есть простая переформулировка (1).

Определение многогранника $\Delta(X)$ можно сделать менее формальным. Можно указать процедуру построения выпуклых многогранников по любым неприводимым проективным многообразиям [9]. При этом многогранники $\Delta(\lambda)$ будут отвечать многообразиям флагов группы $G[10]$.

Я благодарен М. Бриону, В. А. Гинзбургу, Б. Я. Казарновскому, Г. И. Ольшанскому и А. Г. Хованскому за полезные обсуждения.

\title{
ЛИТЕРАТУРА
}

1. Atiyah M. F. Proc. Edinburgh Math. Soc. (2), 26, No. 2, 121-133 (1983). 2. Berenstein A., Zelevinski A. J. Geom. Phys., 5, No. 3 (1989). 3. Brion M. In: Séminaire d'Algèbre Paul Dubreil et Marie-Paul Malliavin. Lect. Notes in Math., Vol. 1296, Springer-Verlag, 1987. 4. Brion M. Duke Math. J., 58, 397-424 (1989). 5. Guillemin V., Sternberg S. Invent. Math., 67, No. 3, 515-538 (1982). 6. Heckmann G. J. Invent. Math., 67, 333-356 (1982). 7. Казарновский Б. Я. Функц. анализ и его прил., 21, вып. 4 (1987). 8. Kouchnirenko A. Invent. Math., 32, 1-31 (1976). 9. Okounkov A. Invent. Math., 125, 405-411 (1996). 10. Okounkov A. to appear in A. A. Kirillov anniversary volume, Adv. Sov. Math., Am. Math. Soc. 11. Хованский А. Г. Функц. анализ и его прил., 26, вып. 4, 57-63 (1992).

Институт проблем передачи информации РАН e-mail: okounkov@ippi.ac.msk.su

Поступило в редакцию 25 декабря 1995 г.

\section{Предельное поведение спектра в одном классе больших случайных матриц}

\author{
(c) 1997. А. Ю. ПлАхов
}

1. Уравнение Марченко-Пастура. В статье В. А. Марченко и Л. А. Пастура [1] рассматривается случайный самосопряженный оператор в $\mathbb{C}^{N}$ вида $A_{N}=A_{0 N}+\sum_{n=1}^{m} \tau_{n}\left(\cdot, q^{n}\right) q^{n}$, где $\tau_{n}$ - действительные независимые случайные величины с одинаковой функцией распределения $\sigma(\tau)$, случайные векторы $q^{n}$ независимы и удовлетворяют некоторым дополнительным условиям, a оператор $A_{0 N}$ неслучаен. Далее, с помощью формулы $\nu_{N}(\lambda)=\{$ число собственных значений $A_{N}$, меньших, чем $\left.\lambda\right\} / N$ определяется нормированная спектральная функция оператора $A_{N}$. Марченко и Пастур доказали, что если (i) $\lim _{N \rightarrow \infty}(m / N)=c$ и (ii) последовательность нормированных спектральных функций операторов $A_{0 N}$ сходится к некоторой монотонно неубывающей функции $\nu_{0}(\lambda)$ во всех ее точках непрерывности, то $\nu_{N}(\lambda)$ сходится по вероятности к неслучайной неубывающей функции $\nu(\lambda)$ во всех ее точках непрерывности и преобразование Стилтьеса этой функщии, $u(z)=\int_{-\infty}^{+\infty}(\lambda-z)^{-1} d \nu(\lambda)$, может быть найдено следующим образом.

* Эта работа выполнена при частичной поддержке фондов INTAS, грант RFBR 95418, RFFI-DFG, грант 96-01-000436. 
Обозначим через $u(z, t)$ решение дифференциального уравнения в частных производных

$$
\partial u(z, t) / \partial t+c \tau(t)(1+\tau(t) u(z, t))^{-1} \partial u(z, t) / \partial z=0
$$

с начальным условием $u(z, 0)=u_{0}(z)$, где $\tau(t)=\inf _{\tau}\{\tau: \sigma(\tau) \geqslant t\}, u_{0}(z)=$ $\int_{-\infty}^{+\infty}(\lambda-z)^{-1} d \nu_{0}(\lambda)$. Тогда $u(z)=u(z, 1)$.

Отметим, что уравнение подобного вида возникает также при изучении безгранично делимых мер в некоммутативной теории вероятностей (см., напримep, [2]).

Марченко и Пастур [1] предложили также следующее простое правило, позволяющее отыскать все интервалы действительной оси, дополнительные к спектру, т. е. интервалы, на которых функция $\nu(\lambda)$ постоянна.

Пусть $I_{0}=\bigcup_{i} I_{0 i} \subset \mathbb{R}$ - объединение максимальных интервалов постоянства функции $\nu_{0}$. На каждом интервале $I_{0 i}$ функция $u_{0}(z)$ действительнозначна и монотонно возрастает; следовательно, существует обратная к ней функция $x_{0 i}(u)$. Введем функцию $x_{i}(u)=x_{0 i}(u)+J(u)$, определенную на множестве $u_{0}\left(I_{0 i}\right) \cap \mathbb{C}_{\sigma}$, и обозначим через $U_{i}$ множество точек $u$, для которых $x_{i}^{\prime}(u)>0$; здесь $J(u)=c \int_{-\infty}^{+\infty} \tau(1+\tau u)^{-1} d \sigma(\tau)$, а $\mathbb{C}_{\sigma}$ обозначает область голоморфности функции $J(u)$. Наконец, обозначим через $I$ объединение интервалов постоянства функции $\nu(\lambda)$.

Правило состоит в следующем: $I=\bigcup_{i} x_{i}\left(U_{i}\right)$.

В настоящей работе дается решение уравнения (1) и приводится схема доказательства правила Марченко-Пастура.

2. Решение уравнения Марченко-Пастура. Введем функцию $f_{t}(w)=$ $w+J_{t}\left(u_{0}(w)\right)$, где $J_{t}(u)=c \int_{-\infty}^{+\infty} \tau(1+\tau u)^{-1} d \sigma_{t}(\tau), \sigma_{t}(\tau)=\min \{\sigma(\tau), t\}$. Мы покажем здесь, что

(I) существует и единственна функция $f_{t}^{-1}: \mathbb{C}^{+} \rightarrow \mathbb{C}^{+}$, такая, что $f_{t}\left(f_{t}^{-1}(z)\right)$ $\equiv z ;$ здесь $\mathbb{C}^{+}=\{z: \operatorname{Im} z>0\}$

(II) $u(z, t)=u_{0}\left(f_{t}^{-1}(z)\right)$.

Набросок доказательства утверждений (I) и (II). Для функций $u_{0}(w)$ и $J_{t}(u)$ справедливы следующие асимптотические оценки:

(a) $u_{0}(w)=o(1), w \rightarrow \infty, \operatorname{Im} w \geqslant v(|w|) ;\left(\right.$ б) $u_{0}(w)=-w^{-1}(1+o(1))$, $w \rightarrow \infty, \operatorname{Im} w \geqslant|w| v(|w|) ;($ в) $\varrho(w) \leqslant 2|w|(1+o(1)), w \rightarrow \infty, \operatorname{Im} w>0$, где $\varrho(w)=2 \operatorname{Im} w\left(|w| \operatorname{Im} u_{0}(w)\right)^{-1} ;(г) J_{t}(u)=o(1 /|u|), u \rightarrow 0, \operatorname{Im} u>$ $|2 u| v(1 /|2 u|)$, с некоторой функщией $v(r)=o(1), r \rightarrow \infty$. Из оценок (б) и (г) выводим, что

$$
J_{t}\left(u_{0}(w)\right) \leqslant \alpha(|w|), \quad w \rightarrow \infty, \operatorname{Im} w \geqslant|w| v(|w|),
$$

где $\alpha(r)=o(r), r \rightarrow+\infty$.

Кроме того, имеют место соотношения

$$
\begin{gathered}
c\left|\operatorname{Re} \int_{|\phi(\tau, w)| \geqslant \varrho(w)} \phi(\tau, w) d \sigma_{t}(\tau)\right| \leqslant-|w|(2 \operatorname{Im} w)^{-1} \operatorname{Im} J_{t}\left(u_{0}(w)\right), \\
\left|\operatorname{Re} \int_{|\phi(\tau, w)|<\varrho(w)} \phi(\tau, w) d \sigma_{t}(\tau)\right|=o(|w|), \quad w \rightarrow \infty, \operatorname{Im} w \geqslant v(|w|),
\end{gathered}
$$


где $\phi(\tau, w)=\tau\left(1+\tau u_{0}(w)\right)^{-1} ;$ последнее соотношение выводится с использованием оценок (а) и (в). Из формул (3) и (4) следует, что

$$
\left|\operatorname{Re} J_{t}\left(u_{0}(w)\right)\right| \leqslant-|w|(2 \operatorname{Im} w)^{-1} \operatorname{Im} J_{t}\left(u_{0}(w)\right)+\alpha_{1}(|w|)
$$

при $w \rightarrow \infty, \operatorname{Im} w \geqslant v(|w|)$, где $\alpha_{1}(r)=o(r), r \rightarrow+\infty$.

Используя неравенства (2) и (5), получаем, что когда $w$ совершает обход в положительном направлении вдоль границы области $B_{R}=\left\{w \in \mathbb{C}^{+}:|w|<R\right.$, $y>v(R)\}$, функция $f_{t}(w)$ совершает ровно один оборот вокруг области $G_{R}$, ограниченной сверху дугой окружности $|z|=R-\alpha(R)$, а снизу - прямыми $\operatorname{Im} z=v(R), \operatorname{Re} z= \pm\left[(2 v(R))^{-1} \operatorname{Im} z+R / 2-\alpha_{2}(R)\right]$, где $\alpha_{2}(R)=\alpha_{1}(R)+$ $R v^{2}(R)$. Согласно принципу аргумента, для любого $z \in G_{R}$ имеется единственное значение $w \in B_{R}$, для которого $f_{t}(w)=z$. Поскольку любая точка из $\mathbb{C}^{+}$ принадлежит всем областям $G_{R}$ и $B_{R}$, начиная с некоторого $R$, мы заключаем, что каждое значение $z \in \mathbb{C}^{+}$имеет единственный прообраз $w=f_{t}^{-1}(z) \in \mathbb{C}^{+}$. Проверка того, что функция $u_{0}\left(f_{t}^{-1}(z)\right)$ является решением уравнения $(1)$, не составляет труда.

3. Схема доказательства правила Марченко-Пастура. Мы докажем здесь равенство $\bigcup_{i}\left\{\left(x_{i}(u), u\right): u \in U_{i}\right\}=\{(x, u(x)): x \in I\}$; правило Марченко-Пастура является его очевидным следствием.

Доказательство прямого включения несложно и использует равенство $x_{i}(u)=$ $f_{1}\left(x_{0 i}(u)\right)$, а также то обстоятельство, что функция, обратная к $x_{i}(u)$, непрерывно продолжается до функции $u_{0}\left(f_{1}^{-1}(z)\right)=u(z)$ на верхней полуокрестности любой точки $x_{i}(u), u \in U_{i}$.

При доказательстве обратного включения нам понадобится следующая лемма, доказательство которой мы здесь опускаем.

Лемма. Пусть $\nu_{0}\left(w_{2}\right)-\nu_{0}\left(w_{1}\right)>0$. Тогда существует значение $w \in$ $\left[w_{1}, w_{2}\right]$, такое, что для любого $\varepsilon>0$ существует $\delta>0$, такое, что для любого $y>0$ либо $\operatorname{Im} u_{0}(w+i y) \geqslant \delta, л и б о ~\left|\operatorname{Re} u_{0}(w+i y)\right|<\varepsilon$.

Пусть $x \in I$; тогда функция $u$ конформно отображает некоторую верхнюю полуокрестность $\mathscr{U}_{+}(x)$ на верхнюю полуокрестность $\mathscr{U}_{+}(u(x))$. Следовательно, функция $u_{0}$ конформно отображает область $f^{-1}\left(\mathscr{U}_{+}(x)\right)$ на $\mathscr{U}_{+}(u(x))$; обозначим через $z_{0}$ обратную к ней функцию.

Рассмотрим сначала случай, когда $u(x) \neq 0$. Выберем в $\mathscr{U}_{+}(u(x))$ последовательности $\left\{u_{n}^{\prime}\right\}$ и $\left\{u_{n}^{\prime \prime}\right\}$, сходящиеся соответственно к действительным точкам $u^{\prime}, u^{\prime \prime}$, таким, что $u^{\prime} u^{\prime \prime}>0$ и $u^{\prime}<u(x)<u^{\prime \prime}$, причем пределы $\lim _{n \rightarrow \infty} z_{0}\left(u_{n}^{\prime}\right)$ $=w^{\prime}, \lim _{n \rightarrow \infty} z_{0}\left(u_{n}^{\prime \prime}\right)=w^{\prime \prime}$ существуют и различны. Возможность такого выбора следует из теоремы Фату (см., например, [3]). Легко видеть, что $w^{\prime}$ и $w^{\prime \prime}$ действительны. Функция $\nu_{0}$ постоянна на $\left(w^{\prime}, w^{\prime \prime}\right)$, так как в противном случае с использованием леммы заключаем, что при некотором $w \in\left(w^{\prime}, w^{\prime \prime}\right)$ и при достаточно больших $n$ кривая $\left\{u_{0}(w+i y), y>0\right\}$ не пересекает отрезков $\left[u_{n}^{\prime}, u_{n}^{\prime \prime}\right]$, в то время как их образы при отображении $z_{0}$ пересекаются. Следовательно, $\left(w^{\prime}, w^{\prime \prime}\right)$ принадлежит некоторому интервалу $I_{0 i}$ и функция $z_{0}(u)$ голоморфно продолжается на интервал $\left(u^{\prime}, u^{\prime \prime}\right)$ и совпадает на нем с $x_{0 i}(u)$.

Далее, функция $f_{1}\left(z_{0}(u)\right)$, обратная к $u(z)$, голоморфно продолжается в окрестность точки $u(x)$ и имеет в этой точке положительную производную. 
Используя формулу

$$
f_{1}\left(z_{0}(u)\right)=z_{0}(u)+J(u),
$$

находим, что $x=x_{0 i}(u)+J(u)$ в точке $u=u(x)$ и функция $J$ голоморфна. Тем самым обратное включение доказано.

В случае $u(x)=0$ из приведенных выше рассуждений следует, что левая и правая окрестности нуля принадлежат $\mathbb{C}_{\sigma}$; отсюда заключаем, что и $0 \in \mathbb{C}_{\sigma}$. Снова используя формулу (6), получаем, что функция $z_{0}(u)$ голоморфно продолжается в окрестность нуля. Дальнейшие рассуждения проводятся так же, как и в предыдущем случае.

\section{ЛитеРАтУРА}

1 Марченко В. А., Пастур Л. А. Матем. сб. 72 (114), №4, 507-536 (1967). 2. Voiculescu D. J. Funct. Anal., 66, 323-346 (1986). 3. Гофман K. Банаховы пространства аналитических функций. ИЛ, М., 1963.

МГТУ им. Н. Э. Баумана

Поступило в редакцию 30 ноября 1995 г.

УдК 519.46

\section{Об одном классе бесконечномерных весовых $\operatorname{sl}(3)$-модулей}

(c) 1997. С. А. Спирин

Пусть $\left\{e_{i}, f_{i}, h_{i}\right\}, i=1,2$, - стандартный набор образующих алгебры Ли $s l(3)=\operatorname{sl}(3, \mathbb{C})$. Будем говорить, что весовой $\operatorname{sl}(3)$-модуль $V$ принадлежит категории $E_{\lambda}$, где $\lambda$ - вес, если 1$)$ множество всех весов модуля $V$ есть $\lambda+\Pi$, где П - решетка, порожденная корнями, и 2) элементы $e_{2}$ и $f_{1}$ действуют на $V$ обратимыми операторами. В работе [1] описана ассоциативная алгебра $Q(s l(3))$, такая, что для любого $\lambda$ категория $E_{\lambda}$ эквивалентна категории $Q(\operatorname{sl}(3))$-mod.

$\mathrm{B}$ настоящей работе для каждого натурального $d$ описано семейство $d$-мерных $Q(s l(3))$-модулей $\left\{Y_{d, \nu}\right\}_{\nu \in \mathbb{C}}$ и для каждого веса $\lambda$ - соответствующее семейство $s l(3)$-модулей $\left\{V_{d, \nu, \lambda}\right\}$ из категории $E_{\lambda}$. Доказано, что всякий конечномерный неприводимый $Q(s l(3))$-модуль изоморфен одному из модулей $Y_{d, \nu}$. Из этого, в частности, следует, что всякий неприводимый $s l(3)$-модуль без кручения и с конечномерными весовыми подпространствами изоморфен одному из модулей $V_{d, \nu, \lambda}$. Кроме того, оказывается, что любой конечномерный неприводимый $s l(3)$-модуль изоморфен подфактору какого-либо из $V_{d, \nu, \lambda}$.

1. $Q(s l(3))$ есть ассоциативная алгебра с единицей, четырьмя образующими $x_{0}, x_{1}, y_{0}, y_{1}$ и соотношениями

$$
\begin{aligned}
x_{0} y_{0}-y_{0} x_{0}=x_{1} y_{0}+y_{1} x_{0}, & x_{1} y_{1}-y_{1} x_{1}=x_{1}+y_{1}, \\
y_{0} x_{1}-x_{1} y_{0}=-y_{1} x_{1}-x_{0}, & x_{0} y_{1}-y_{1} x_{0}=x_{1} y_{1}+y_{0}, \\
x_{0} x_{1}-x_{1} x_{0}=-x_{1}^{2}+x_{0}, & y_{0} y_{1}-y_{1} y_{0}=y_{1}^{2}-y_{0} .
\end{aligned}
$$

